\title{
Two Efficient Bi-Parametric Derivative Free With Memory Methods for Finding Simple Roots Nonlinear Equations
}

\author{
J. P. Jaiswal \\ Department of Mathematics, Maulana Azad National Institute of Technology, Bhopal, M.P., India-462003 \\ Email: asstprofjpmanit@gmail.com
}

\begin{abstract}
The present paper is devoted to the improvement of the existing fourth-and eighth-order derivative free methods without memory proposed by Cordero et al. (2013). To achieve this goal two parameters are introduced which are calculated with the help of Newton's interpolatory polynomial. It is shown that the $R$-order convergence of the proposed methods has been increased from 4 to 7 and 8 to 14 , respectively without any extra evaluation. Two non-smooth examples are demonstrated to confirm theoretical results. Numerically the modified methods are examined along with comparison to recent existing with memory methods.
\end{abstract}

Keywords: Derivative free method, nonlinear equations, order of convergence, efficiency index, nonsmooth function.

\section{Introduction}

The main motive in constructing iterative algorithms for solving nonlinear equations is to achieve as high as possible convergence rate with a fixed number of function evaluations per iteration. In this work we study the multipoint methods with memory, a work which is very rarely discussed in the literature in spite of high computational efficiency of this kind of root-finding methods. Most of these methods are improvement of multipoint methods without memory with optimal order of convergence. Using Newton's interpolation with divided difference, deadly fast convergence of new methods with memory is attained without adding more function evaluations. As a result, these multipoint methods hold a very high computational efficiency. Let $p_{k}$ represent the $m+1$ quantities $x_{k}, t_{1}\left(x_{k}\right), t_{2}\left(x_{k}\right), \ldots, t_{m}\left(x_{k}\right), k \geq 1$ and define an iterative process by

$$
x_{k+1}=F\left(p_{k} ; p_{k-1}, p_{k-2}, \ldots, p_{k-m}\right) .
$$

Following Traub's terminology [10], $F$ is called a multipoint iteration function with memory. Probably Traub initiated the idea of with memory method in his book [10]. For this he considered Steffensen type method

$$
x_{k+1}=x_{k}-\frac{\gamma f\left(x_{k}\right)^{2}}{f\left(x_{k}+\gamma f\left(x_{k}\right)\right)-f\left(x_{k}\right)},
$$

where $\gamma$ is arbitrary parameter. This method has quadratic convergence. To compare iterative methods theoretically, Owtrowski [1] introduced the idea of efficiency index given by $r^{1 / \theta_{f}}$, where $r$ is the order of convergence and $\theta_{f}$ number of function evaluations per iteration. In other words we can say that an iterative method with higher efficiency index is more efficient. To accelerate the convergence order of this method without using additional evaluation $\gamma$ is recursively calculated by self-accelerating method. Let $\gamma_{0}$ be the given initial parameter and consider

$$
\begin{aligned}
\phi_{k} & =\frac{f\left(x_{k}+\gamma_{k} f\left(x_{k}\right)\right)-f\left(x_{k}\right)}{\gamma_{k} f\left(x_{k}\right)}, k=0,1,2, \ldots, \\
x_{k+1} & =x_{k}-\frac{f\left(x_{k}\right)}{\phi_{k}} .
\end{aligned}
$$


where

$$
\gamma_{k}=-\frac{1}{\phi_{k-1}}, k=1,2, \ldots
$$

Traub derived that order of convergence of this method is 2.414. And thus the order of convergence of (2) with memory is more than that of Steffensen method, which also needs two function evaluations per iteration. Motivated by this currently researchers are trying to increase the efficiency of the existing optimal order without memory methods by using single or double parameters. In the literature with memory methods with two parameters are very rare.

In the present paper we present an improvement of the existing optimal fourth-and eighth-order derivative free method constructed by introducing two self accelerating parameters. These parameters are calculated with the help of Newton's interpolatory polynomial. In section 2, derivative free twoand three-points methods with memory with improved order of convergence from 4 to 7 and 8 to 14 , respectively without extra evaluations are presented. Two non-smooth equations have been considered to give the comparisons of absolute errors and computational efficiencies are given in section 3 to illustrate convergence behavior. Finally, we give the concluding remark.

\section{Development and Construction of With Memory Method}

In the convergence analysis of the new method, we employ the notation used in Traub's book [10]: if $m_{k}$ and $n_{k}$ are null sequences and $m_{k} / n_{k} \rightarrow C$, where $\mathrm{C}$ is a non-zero constant, we shall write $m_{k}=O\left(n_{k}\right)$ or $m_{k} \sim C n_{k}$. We also use the concept of $R$-order of convergence introduced by Ortega and Rheinboldt [11]. Let $x_{k}$ be a sequence of approximations generated by an iterative method (IM). If this sequence converges to a zero $\xi$ of function $f$ with the $R$-order $O_{R}((I M), \xi) \geq r$, we will write

$$
e_{k+1} \sim A_{k, r} e_{k}^{r}
$$

where $A_{k, r}$ tends to the asymptotic error constant $A_{r}$ of the iterative method (IM) when $k \rightarrow \infty$.

Very recently Cordero et al. [2] presented derivative-free optimal fourth- and eighth-order iterative methods as follows:

For given $x_{0}$, consider

$$
\begin{aligned}
z_{n} & =x_{n}+f\left(x_{n}\right), n=0,1,2, \ldots, \\
y_{n} & =x_{n}-\frac{f\left(x_{n}\right)}{f\left[x_{n}, z_{n}\right]}, \\
x_{n+1} & =y_{n}-\frac{f\left(y_{n}\right) f\left[x_{n}, z_{n}\right]}{f\left[x_{n}, y_{n}\right] f\left[y_{n}, z_{n}\right]}
\end{aligned}
$$

and

$$
\begin{aligned}
z_{n} & =x_{n}+f\left(x_{n}\right), n=0,1,2, \ldots, \\
y_{n} & =x_{n}-\frac{f\left(x_{n}\right)}{f\left[x_{n}, z_{n}\right]}, \\
u_{n} & =y_{n}-\frac{f\left(y_{n}\right) f\left[x_{n}, z_{n}\right]}{f\left[x_{n}, y_{n}\right] f\left[y_{n}, z_{n}\right]}, \\
x_{n+1} & =u_{n}-\frac{f\left(u_{n}\right)}{b_{2}-b_{1} b_{4}},
\end{aligned}
$$

where

$$
\begin{gathered}
b_{4}=\frac{f\left[y_{n}, u_{n}, x_{n}\right]-f\left[y_{n}, u_{n}, z_{n}\right]}{f\left[y_{n}, z_{n}\right]-f\left[y_{n}, x_{n}\right]}, \\
b_{3}=f\left[y_{n}, u_{n}, z_{n}\right]+b_{4} f\left[y_{n}, z_{n}\right],
\end{gathered}
$$




$$
\begin{gathered}
b_{2}=f\left[y_{n}, u_{n}\right]-b_{3}\left(y_{n}-u_{n}\right)+f\left(y_{n}\right) b_{4}, \\
b_{1}=f\left(u_{n}\right) .
\end{gathered}
$$

If we introduce two different parameters in $z_{n}$ and $y_{n}$ involved in the above methods then the modified methods are given by along with its error expressions as follows:

Modified method I. For suitably given $x_{0}$,

$$
\begin{aligned}
z_{n} & =x_{n}+\epsilon f\left(x_{n}\right), n=0,1,2, \ldots, \\
y_{n} & =x_{n}-\frac{f\left(x_{n}\right)}{f\left[x_{n}, z_{n}\right]+\delta f\left(z_{n}\right)}, \\
x_{n+1} & =y_{n}-\frac{f\left(y_{n}\right) f\left[x_{n}, z_{n}\right]}{f\left[x_{n}, y_{n}\right] f\left[y_{n}, z_{n}\right]},
\end{aligned}
$$

and its error expression is given by

$$
e_{n+1}=\frac{\left(1+\epsilon c_{1}\right)^{2}\left(\delta c_{1}+c_{2}\right)\left(2 c_{2}^{2}+c_{1}\left(\delta c_{2}-c_{3}\right)\right)}{c_{1}^{3}} e_{n}^{4}+O\left(e_{n}^{5}\right) .
$$

Modified method II. For suitably given $x_{0}$,

$$
\begin{aligned}
z_{n} & =x_{n}+\alpha f\left(x_{n}\right), n=0,1,2, \ldots, \\
y_{n} & =x_{n}-\frac{f\left(x_{n}\right)}{f\left[x_{n}, z_{n}\right]+\beta f\left(z_{n}\right)}, \\
u_{n} & =y_{n}-\frac{f\left(y_{n}\right) f\left[x_{n}, z_{n}\right]}{f\left[x_{n}, y_{n}\right] f\left[y_{n}, z_{n}\right]}, \\
x_{n+1} & =u_{n}-\frac{f\left(u_{n}\right)}{b_{2}-b_{1} b_{4}},
\end{aligned}
$$

and its error expression is given by

$$
\begin{aligned}
e_{n+1}= & \frac{\left(1+\alpha c_{1}\right)^{4}\left(\beta c_{1}+c_{2}\right)^{2}\left(2 c_{2}^{2}+c_{1}\left(\beta c_{2}-c_{3}\right)\right)\left(2 c_{2}^{4}+c_{1} c_{2}^{2}\left(\beta c_{2}-c_{3}\right)-c_{1}^{2} c_{3}^{2}+c_{1}^{2} c_{2} c_{4}\right)}{c_{1}^{7} c_{2}} e_{n}^{8}, \\
& +O\left(e_{n}^{9}\right),
\end{aligned}
$$

where $b_{1}, b_{2}, b_{3}, b_{4}$ are as previously defined and $c_{i}=\frac{f^{(i)}(\xi)}{i !}$. Since the above error equations contain the parameters, which can be approximated in such a way that increases the local convergence order. For this purpose, first we put $\epsilon=\epsilon_{k}, \delta=\delta_{k}$ and $\alpha=\alpha_{k}, \beta=\beta_{k}$ and then approximations of these parameters are given by

$$
\begin{aligned}
\epsilon_{k} & =-\frac{1}{c_{1}} \approx-\frac{1}{\widetilde{c_{1}}}=-\frac{1}{N_{3}^{\prime}\left(x_{k}\right)}, \\
\delta_{k} & =-\frac{c_{2}}{c_{1}} \approx-\frac{\overline{c_{2}}}{\overline{c_{1}}}=-\frac{N_{4}^{\prime \prime}\left(w_{k}\right)}{2 N_{4}^{\prime}\left(w_{k}\right)} .
\end{aligned}
$$

and

$$
\begin{aligned}
& \alpha_{k}=-\frac{1}{c_{1}} \approx-\frac{1}{\tilde{c_{1}}}=-\frac{1}{\tilde{N}_{4}^{\prime}\left(x_{k}\right)}, \\
& \beta_{k}=-\frac{c_{2}}{c_{1}} \approx-\overbrace{\frac{\overbrace{c_{2}}}{c_{1}}}=-\frac{\tilde{N}_{5}^{\prime \prime}\left(w_{k}\right)}{2 \tilde{N}_{5}^{\prime}\left(w_{k}\right)} .
\end{aligned}
$$

where

$N_{3}(t)=N_{3}\left(t ; x_{k}, y_{k-1}, x_{k-1}, z_{k-1}\right), N_{4}(t)=N_{4}\left(t ; x_{k}, w_{k}, y_{k-1}, x_{k-1}, z_{k-1}\right)$ and

$\tilde{N}_{4}(t)=\tilde{N}_{4}\left(t ; x_{k}, u_{k-1}, y_{k-1}, x_{k-1}, z_{k-1}\right), \tilde{N}_{5}(t)=\tilde{N}_{5}\left(t ; x_{k}, w_{k}, u_{k-1}, y_{k-1}, x_{k-1}, z_{k-1}\right)$

are the Newton's interpolatory polynomial of degree three, four and five respectively. Before going to prove the main result, we state the following two lemmas which can be obtained by using the error of Newton's interpolation, in the same manner as in [3]. 
Lemma 1. If $\epsilon_{k}=-\frac{1}{N_{3}^{\prime}\left(x_{k}\right)}$ and $\delta_{k}=-\frac{N_{4}^{\prime}\left(w_{k}\right)}{2 N_{4}^{\prime}\left(w_{k}\right)}$, then the estimates

$$
\begin{aligned}
\text { (i) } 1+\epsilon_{k} c_{1} & \sim \frac{c_{4}}{c_{1}} e_{k-1, y} e_{k-1, z} e_{k-1}, \\
\text { (ii) } \delta_{k} c_{1}+c_{2} & \sim-c_{5} e_{k-1, y} e_{k-1, z} e_{k-1} .
\end{aligned}
$$

Lemma 2. If $\alpha_{k}=-\frac{1}{\tilde{N}_{4}^{\prime}\left(x_{k}\right)}$ and $\beta_{k}=-\frac{\tilde{N}_{5}^{\prime}\left(w_{k}\right)}{2 \tilde{N}_{5}^{\prime}\left(w_{k}\right)}$, then the estimates

$$
\begin{aligned}
\text { (i) } 1+\alpha_{k} c_{1} & \sim-\frac{c_{5}}{c_{1}} e_{k-1, u} e_{k-1, y} e_{k-1, z} e_{k-1}, \\
\text { (ii) } \beta_{k} c_{1}+c_{2} & \sim c_{6} e_{k-1, u} e_{k-1, y} e_{k-1, z} e_{k-1}
\end{aligned}
$$

The theoretical proof of the order of convergence of the proposed methods is given by the following theorem:

Theorem 1. If an initial approximation $x_{0}$ is sufficiently close to a simple zero $\xi$ of $f(x)$ and the parameters $\epsilon_{k}, \delta_{k}$ and $\alpha_{k}, \beta_{k}$ in the iterative scheme (6) and (8) are recursively calculated by the forms given in (10) and (11), respectively. Then the $R$-order of convergence of with memory schemes (6) and (8) is at least seven and fourteen, respectively.

Proof. First, we assume that the $R$-order of convergence of the sequence $x_{k}, z_{k}, y_{k}, u_{k}$ is at least $m, m_{1}$, $m_{2}$ and $m_{3}$, respectively. Hence

$$
e_{k+1} \sim A_{k, m} e_{k}^{m} \sim A_{k, m}\left(A_{k-1, m} e_{k-1}^{m}\right)^{m} \sim A_{k, m} A_{k-1, m}^{m} e_{k-1}^{m^{2}} .
$$

and

$$
e_{k, z} \sim A_{k, m_{1}} e_{k}^{m_{1}} \sim A_{k, m_{1}}\left(A_{k-1, m} e_{k-1}^{m}\right)^{m_{1}} \sim A_{k, m_{1}} A_{k-1, m}^{m_{1}} e_{k-1}^{m m_{1}}
$$

Similarly

$$
\begin{aligned}
& e_{k, y} \sim A_{k, m_{2}} A_{k-1, m}^{m_{2}} e_{k-1}^{m m_{2}}, \\
& e_{k, u} \sim A_{k, m_{3}} A_{k-1, m}^{m_{3}} e_{k-1}^{m m_{3}} .
\end{aligned}
$$

Now we will prove the results in two parts. First for method (6) and then for (8).

Modified method I. For method (6), it can be derived that

$$
\begin{gathered}
e_{k, z} \sim\left(1+\epsilon_{k} c_{1}\right) e_{k}, \\
e_{k, y} \sim L_{1}\left(1+\epsilon_{k} c_{1}\right)\left(\delta_{k} c_{1}+c_{2}\right) e_{k}^{2}, \text { where } L_{1}=\frac{1}{c_{1}}, \\
e_{k+1} \sim L_{2}\left(1+\epsilon_{k} c_{1}\right)^{2}\left(\delta_{k} c_{1}+c_{2}\right) e_{k}^{4}, \text { where } L_{2}=\frac{c_{2}\left(2 c_{2}^{2}+c_{1}\left(\delta_{k} c_{2}-c_{3}\right)\right)}{c_{1}^{3}} .
\end{gathered}
$$

Using the results of lemma (2.1) in the equations (16), (17) and (18), we have

$$
\begin{gathered}
e_{k, z} \sim \frac{c_{4}}{c_{1}}\left(A_{k-1, m_{2}}\right)\left(A_{k-1, m_{1}}\right)\left(A_{k-1, m}\right) e_{k-1}^{m_{2}+m_{1}+m+1}, \\
e_{k, y} \sim-\frac{c_{4} c_{5}}{c_{1}} L_{1}\left(A_{k-1, m_{2}}^{2}\right)\left(A_{k-1, m_{1}}^{2}\right)\left(A_{k-1 m}^{2}\right) e_{k-1}^{2 m_{2}+2 m_{1}+2 m+2},
\end{gathered}
$$


and

$$
e_{k+1} \sim-\frac{c_{4}^{2} c_{5}}{c_{1}^{2}} L_{2}\left(A_{k-1, m_{2}}^{3}\right)\left(A_{k-1, m_{1}}^{3}\right)\left(A_{k-1, m}^{4}\right) e_{k-1}^{3 m_{2}+3 m_{1}+4 m+3}
$$

Now comparing the equal powers of $e_{k-1}$ in (13)-(19); (14)- (20) and (12)- (21), we get the following nonlinear system

$$
\begin{aligned}
m m_{1}-m_{2}-m_{1}-m-1 & =0, \\
m m_{2}-2 m_{2}-2 m_{1}-2 m-2 & =0, \\
m^{2}-3 m_{2}-3 m_{1}-4 m-3 & =0 .
\end{aligned}
$$

After solving these equations, we get $m=7, m_{2}=4, m_{1}=2$. It confirms the convergence of method (6). This shows the first part.

Modified method II. For method (8), it can be derived that

$$
\begin{gathered}
e_{k, z} \sim\left(1+\alpha_{k} c_{1}\right) e_{k}, \\
e_{k, y} \sim L_{1}\left(1+\alpha_{k} c_{1}\right)\left(\beta_{k} c_{1}+c_{2}\right) e_{k}^{2}, \text { where } L_{1}=\frac{1}{c_{1}}, \\
e_{k, u} \sim O_{1}\left(1+\alpha_{k} c_{1}\right)^{2}\left(\beta_{k} c_{1}+c_{2}\right) e_{k}^{4},
\end{gathered}
$$

where $O_{1}=\frac{c_{2}\left(2 c_{2}^{2}+c_{1}\left(\beta_{k} c_{2}-c_{3}\right)\right)}{c_{1}^{3}}$ and

$$
e_{k+1} \sim O_{2}\left(1+\alpha_{k} c_{1}\right)^{4}\left(\beta_{k} c_{1}+c_{2}\right)^{2} e_{k}^{8},
$$

where $O_{2}=\frac{\left(2 c_{2}^{2}+c_{1}\left(\beta_{k} c_{2}-c_{3}\right)\right)\left(2 c_{2}^{4}+c_{1} c_{1}^{2}\left(\beta_{k} c_{2}-c_{3}\right)+c_{1}^{2}\left(-c_{3}^{2}+c_{2} c_{4}\right)\right)}{c_{1}^{7} c_{2}}$. Now using the results of lemma (2.2) in the equations (22), (23), (24) and (25), we have

$$
\begin{gathered}
e_{k, z} \sim-\frac{c_{5}}{c_{1}}\left(A_{k-1, m_{3}}\right)\left(A_{k-1, m_{2}}\right)\left(A_{k-1, m_{1}}\right)\left(A_{k-1, m}\right) e_{k-1}^{m_{3}+m_{2}+m_{1}+m+1}, \\
e_{k, y} \sim-\frac{c_{5} c_{6}}{c_{1}} L_{1}\left(A_{k-1, m_{3}}^{2}\right)\left(A_{k-1, m_{2}}^{2}\right)\left(A_{k-1, m_{1}}^{2}\right)\left(A_{k-1 m}^{2}\right) e_{k-1}^{2 m_{3}+2 m_{2}+2 m_{1}+2 m+2}, \\
e_{k, u} \sim\left(\frac{c_{5}^{2} c_{6}}{c_{1}^{2}}\right) O_{1}\left(A_{k-1, m_{3}}^{3}\right)\left(A_{k-1, m_{2}}^{3}\right)\left(A_{k-1, m_{1}}^{3}\right)\left(A_{k-1, m}^{4}\right) e_{k-1}^{3 m_{3}+3 m_{2}+3 m_{1}+4 m+3} .
\end{gathered}
$$

and

$$
e_{k+1} \sim\left(\frac{c_{5}^{4} c_{6}^{2}}{c_{1}^{4}}\right) O_{2}\left(A_{k-1, m_{3}}^{6}\right)\left(A_{k-1, m_{2}}^{6}\right)\left(A_{k-1, m_{1}}^{6}\right)\left(A_{k-1, m}^{8}\right) e_{k-1}^{6 m_{3}+6 m_{2}+6 m_{1}+8 m+6}
$$

Comparing the equal powers of $e_{k-1}$ in (13)-(26); (14)- (27); (16)- (28) and (12)- (29), we get the following nonlinear system

$$
\begin{aligned}
m_{1}-m_{3}-m_{2}-m_{1}-m-1 & =0, \\
m m_{2}-2 m_{3}-2 m_{2}-2 m_{1}-2 m-2 & =0, \\
m m_{3}-3 m_{3}-3 m_{2}-3 m_{1}-4 m-3 & =0, \\
m^{2}-6 m_{3}-6 m_{2}-6 m_{1}-8 m-6 & =0 .
\end{aligned}
$$


After solving these equations we get $m=14, m_{3}=7, m_{2}=4, m_{1}=2$. And thus proof is completed.

Note 1.: The efficiency index of the proposed method (6) along with (10) is $7^{1 / 3}=1.9129$ which is more than $4^{1 / 3}=1.5874$ of method (4).

Note 2.: The efficiency index of the proposed method (8) along with (11) is $14^{1 / 4}=1.9343$ which is more than $8^{1 / 4}=1.6818$ of method $(5)$.

\section{Application to Non-smooth Equations}

In this section we are going to check the effectiveness of the new with memory methods (6) with (10) (NMWM7) and (8) with (12) (NMWM14), comparing them with some recent established with memory methods. Specifically, we consider the sixth-order method (JMWM6) introduced by Jovana in [3], sixthorder method (LMWM6) introduced by Lotfi et al. in [4], seventh-order method (CMWM7) introduced by Cordero et al. in [5], twelfth-order method I (LTMWM12I), II (LTMWM12II), III (LTMWM12III) and IV (LTMWM12IV) introduced by Lotfi and Tavakoli in [6], twelfth-order method I (EMWM12I), II (EMWM12II) and III (EMWM12III) introduced by Eftekhari in [7] and fourteenth-order I (LMWM14I) and II (LMWM14II) introduced by Lotfi et al. in [8]. Nowadays, high-order methods are important because numerical applications use high precision in their computations; for this reason numerical tests have been carried out using variable precision arithmetic in MATHEMATICA 8 with 100 significant digits. Tables 1 and 2 show the absolute error for the first, second and third iterations. To check the theoretical order of convergence, we calculate the computational order of convergence (COC) using the following formula:

$$
C O C=\frac{\ln \left(\left|f\left(x_{n}\right) / f\left(x_{n-1}\right)\right|\right)}{\ln \left(\left|f\left(x_{n-1}\right) / f\left(x_{n-2}\right)\right|\right)}
$$

We test the performances of new methods for the following two non-smooth functions:

$$
\begin{aligned}
& \text { 1. } f_{1}(x)=10\left(x^{4}+x\right), x<0 \\
& =-10\left(x^{3}+x\right), x \geq 0 . \\
& \text { 2. } f_{2}(x)=x(x+1), x<0 \\
& =-2 x(x-1), x \geq 0 \text {. }
\end{aligned}
$$

Table 1. Numerical results for $f_{1}(x)$.

\begin{tabular}{ccccc}
\hline Method & $\left|x_{1}-\xi\right|$ & $\left|x_{2}-\xi\right|$ & $\left|x_{3}-\xi\right|$ & COC \\
\hline & $x_{0}=-0.8$, & $\gamma_{0}=0.01$, & $\alpha_{0}=0.01$, & $\xi=-1$ \\
\hline JMWM6 & $0.15732 \mathrm{e}+0$ & $0.36669 \mathrm{e}-5$ & $0.34403 \mathrm{e}-37$ & 6.7250 \\
LMWM6 & $0.16036 \mathrm{e}+0$ & $0.25566 \mathrm{e}-3$ & $0.15859 \mathrm{e}-19$ & 5.5324 \\
CMWM7 & $0.14660 \mathrm{e}-1$ & $0.10220 \mathrm{e}-11$ & $0.62905 \mathrm{e}-83$ & 7.0025 \\
NMWM7 & $0.49246 \mathrm{e}-1$ & $0.78791 \mathrm{e}-8$ & $0.30306 \mathrm{e}-55$ & 6.9341 \\
LTMWM12I & $0.18233 \mathrm{e}+1$ & $0.99985 \mathrm{e}+0$ & $1.00000 \mathrm{e}+0$ & 2.2130 \\
LTMWM12II & $0.86418 \mathrm{e}-1$ & $0.52042 \mathrm{e}-9$ & $0.00000 \mathrm{e}+0$ & 11.824 \\
LTMWM12III & $0.12019 \mathrm{e}-1$ & $0.36933 \mathrm{e}-6$ & $0.00000 \mathrm{e}+0$ & 12.343 \\
LTMWM12IV & $0.36954 \mathrm{e}+0$ & $0.36954 \mathrm{e}+0$ & $0.36954 \mathrm{e}+0$ & 1.0042 \\
EMWM12I & $0.57265 \mathrm{e}-1$ & $0.10604 \mathrm{e}-9$ & $0.00000 \mathrm{e}+0$ & 11.117 \\
EMWM12II & $0.14034 \mathrm{e}+2$ & $0.47011 \mathrm{e}+1$ & $0.11965 \mathrm{e}+1$ & 1.0065 \\
EMWM12III & $0.57265 \mathrm{e}-1$ & $0.10604 \mathrm{e}-9$ & $0.00000 \mathrm{e}+0$ & 11.117 \\
LMWM14I & $0.41895 \mathrm{e}-3$ & $0.00000 \mathrm{e}+0$ & $0.00000 \mathrm{e}+0$ & 14.001 \\
LMWM14II & $0.32328 \mathrm{e}-2$ & $0.00000 \mathrm{e}+0$ & $0.00000 \mathrm{e}+0$ & 13.993 \\
NMWM14 & $0.41987 \mathrm{e}-2$ & $0.00000 \mathrm{e}+0$ & $0.00000 \mathrm{e}+0$ & 13.998 \\
\hline
\end{tabular}


Table 2. Numerical results for $f_{2}(x)$.

\begin{tabular}{ccccc}
\hline Method & $\left|x_{1}-\xi\right|$ & $\left|x_{2}-\xi\right|$ & $\left|x_{3}-\xi\right|$ & COC \\
\hline & $x_{0}=0.1$, & $\gamma_{0}=0.01$, & $\alpha_{0}=0.01$, & $\xi=0$ \\
\hline JMWM6 & $0.58358 \mathrm{e}-2$ & $0.13033 \mathrm{e}-9$ & $0.72402 \mathrm{e}-11$ & 0.2118 \\
LMWM6 & $0.58908 \mathrm{e}-2$ & $0.27816 \mathrm{e}-5$ & $0.25682 \mathrm{e}-11$ & 1.8159 \\
CMWM7 & $0.57743 \mathrm{e}-2$ & $0.72552 \mathrm{e}-10$ & $0.48367 \mathrm{e}-11$ & 0.1944 \\
NMWM7 & $0.58035 \mathrm{e}-2$ & $0.50597 \mathrm{e}-10$ & $0.24093 \mathrm{e}-11$ & 0.2093 \\
LTMWM12I & $0.47739 \mathrm{e}-3$ & $0.10092 \mathrm{e}-8$ & $0.70720 \mathrm{e}-19$ & 1.7894 \\
LTMWM12II & $0.49877 \mathrm{e}-3$ & $0.10930 \mathrm{e}-8$ & $0.82960 \mathrm{e}-19$ & 1.7882 \\
LTMWM12III & $0.49306 \mathrm{e}-3$ & $0.10704 \mathrm{e}-8$ & $0.79560 \mathrm{e}-19$ & 1.7886 \\
LTMWM12IV & $0.48311 \mathrm{e}-3$ & $0.10313 \mathrm{e}-8$ & $0.73864 \mathrm{e}-19$ & 1.7891 \\
EMWM12I & $0.26019 \mathrm{e}-3$ & $0.28620 \mathrm{e}-9$ & $0.54599 \mathrm{e}-20$ & 1.7990 \\
EMWM12II & $0.26008 \mathrm{e}-3$ & $0.28586 \mathrm{e}-9$ & $0.54466 \mathrm{e}-20$ & 1.7990 \\
EMWM12III & $0.26019 \mathrm{e}-3$ & $0.28620 \mathrm{e}-9$ & $0.54599 \mathrm{e}-20$ & 1.7990 \\
LMWM14I & $0.46693 \mathrm{e}-3$ & $0.10356 \mathrm{e}-35$ & $0.71493 \mathrm{e}-73$ & 1.1380 \\
LMWM14II & $0.44276 \mathrm{e}-3$ & $0.26703 \mathrm{e}-36$ & $0.47536 \mathrm{e}-74$ & 1.1364 \\
NMWM14 & $0.29387 \mathrm{e}-2$ & $0.15456 \mathrm{e}-8$ & $0.14567 \mathrm{e}-87$ & 11.966 \\
\hline
\end{tabular}

\section{Summary}

In this study, convergence order of the existing fourth-and eighth-order derivative free methods has been improved without any extra evaluation. As a result the efficiency is also increased. To justify the theoretical convergence order two non-smooth functions are presented. The numerical results show that proposed method is very useful to find an acceptable approximation of the exact solution of nonlinear equations, specially when the function is non-differentiable.

\section{Acknowledgment}

The author would like to pay his gratitude to the reviewers and editor for their valuable comments.

\section{References}

1. A. M. Owtrowski: Solution of equations and systems of equations, Academic Press, New York (1960).

2. A. Cordero; J. L. Huesoa; E. MartÃnnez and J. R. Torregrosa: A new technique to obtain derivative-free optimal iterative methods for solving nonlinear equations, Journal of Computational and Applied Mathematics 252 (2013), 95-102.

3. J. Dzunic: On efficient two-parameter methods for solving nonlinear equations, Numer. Algor. (2013) 63, 549-569.

4. T. Lotfi, S. Shateyi and S. Hadadi: Potra-Ptak iterative method with memory, ISRN Mathematical Analysis, Volume 2014, Article ID 697642, 6 pages.

5. A. Cordero, T. Lotfi and P. Bakhtiariand J. R. Torregrosa: An efficient two-parametric family with memory for nonlinear equations, Numer. Algor. DOI 10.1007/s11075-014-9846-8.

6. T. Lotfi and E. Tavakoli: On a new efficient Steffenssen-like iterative class by applying a suitable self-accelerator parameter, The Scientific World Journal, Volume 2014, Article ID 769758, 9 pages.

7. T. Eftekhari: On some iterative methods with memory and high efficiency index for solving nonlinear equations, International Journal of Differential Equations, Volume 2014, Article ID 495357, 6 pages.

8. T. Lotfi, F. Soleymani, S. Shateyi, P. Assari, and F. K. Haghani: New mono- and bi-accelerator iterative methods with memory for nonlinear equations, Abstract and Applied Analysis, Volume 2014, Article ID 705674, 8 pages.

9. M. A. Hafiz and Mohamed S. M. Bhagat: Solving non-smooth equations using family of derivative-free optimal methods, Journal of the Egyptian Mathematical Society (2013) 21, 38-43.

10. J. F. Traub: Iterative methods for the solution of equations, Prentice-Hall, Englewood Cliffs, New Jersey (1964). 
11. J. M. Ortega and W. C. Rheinboldt: Iterative solution of nonlinear equations in several variables, Academic Press, New York (1970).

12. J. P. Jaiswal: Two bi-accelerator improved with memory schemes for solving nonlinear equations, Discrete Dynamics in Nature and Society, Volume 2015, Article ID 938606, 7 pages. 